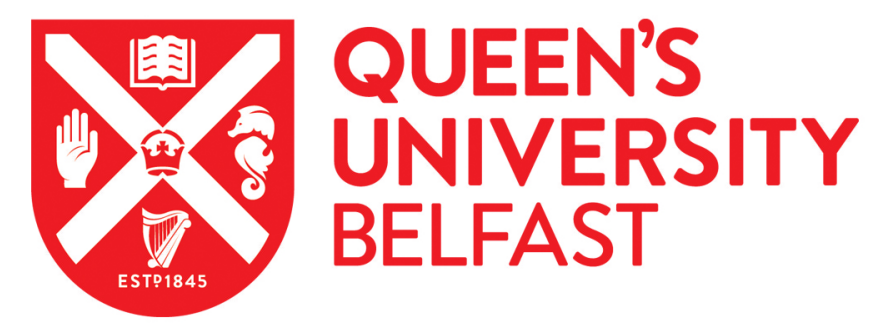

\title{
Helminths, polyparasitism, and the gut microbiome in the Philippines
}

Gordon, C. A., Krause, L., McManus, D. P., Morrison, M., Weerakoon, K. G., Connor, M. C., Olveda, R. M., Ross, A. G., \& Gobert, G. N. (2020). Helminths, polyparasitism, and the gut microbiome in the Philippines. International Journal for Parasitology, 50(3), 217-225. https://doi.org/10.1016/j.ijpara.2019.12.008

\section{Published in:}

International Journal for Parasitology

\section{Document Version:}

Peer reviewed version

Queen's University Belfast - Research Portal:

Link to publication record in Queen's University Belfast Research Portal

\footnotetext{
General rights

Copyright for the publications made accessible via the Queen's University Belfast Research Portal is retained by the author(s) and / or other copyright owners and it is a condition of accessing these publications that users recognise and abide by the legal requirements associated with these rights.

Take down policy

The Research Portal is Queen's institutional repository that provides access to Queen's research output. Every effort has been made to ensure that content in the Research Portal does not infringe any person's rights, or applicable UK laws. If you discover content in the Research Portal that you believe breaches copyright or violates any law, please contact openaccess@qub.ac.uk.
} 


\section{Helminths, polyparasitism, and the gut microbiome in the Philippines}

2 Catherine A. Gordon ${ }^{\text {a }}$, Lutz Krause ${ }^{\mathrm{b}}$, Donald P. McManus ${ }^{\mathrm{a}}$, Mark Morrison ${ }^{\mathrm{b}}$, Kosala G. Weerakoon

3 a,c,d, Mairead C. Connor ${ }^{\text {e }}$, Remigio M. Olveda ${ }^{\text {f }}$, Allen G. Ross ${ }^{\text {g }}$, Geoffrey N. Gobert ${ }^{\text {e,* }}$

$4 \quad$ a Molecular Parasitology Laboratory, Immunology Division, QIMR Berghofer Medical Research

5 Institute, Brisbane, Australia

6 b University of Queensland Diamantina Institute, Faculty of Medicine, Translational Research

7 Institute, Brisbane, Australia

$8{ }^{\mathrm{c}}$ School of Public Health, University of Queensland, Brisbane, Australia

9 d Department of Parasitology, Faculty of Medicine and Allied Sciences, Rajarata University of Sri

10 Lanka, Saliyapura, Sri Lanka

11 e School of Biological Sciences, Queen's University Belfast, Belfast, United Kingdom

$12{ }^{\mathrm{f}}$ Department of Immunology, Research Institute of Tropical Medicine, Manilla, the Philippines

$13{ }^{\mathrm{g}}$ Menzies Health Institute Queensland, Gold Coast, Australia

14 *Corresponding author. Geoffrey Gobert. E-mail address: g.gobert@qub.ac.uk 
Polyparasitism, involving soil-transmitted helminths (STH) and Schistosoma blood flukes, is

18 common in low to middle income countries. These helminths impact on the gut environment and can

19 cause changes to the gut microbiome composition. Here we examined the gut microbiome in 20 individuals with polyparasitism from two human cohorts in the Philippines utilising DNA 21 sequencing-based profiling. Multiple helminth species infections were high with $70.3 \%$ of study 22 participants harbouring at least two parasite species, and 16\% harbouring at least five species. 23 Increased numbers of helminth co-infections, in particular with the gut-resident STH, were 24 significantly associated with increased bacterial diversity; however no significant parasite-gut 25 microbiome associations were evident for individuals infected only with Schistosoma japonicum. In general, a healthy gut is associated with high bacterial diversity, which in these human cohorts may be the result of helminth-mediated immune modulation, or due to changes in the gut environment caused by these parasitic helminths.

Keywords: Soil-transmitted helminths; Hookworm; Ancylostoma; Ascaris; Trichuris; Schistosoma;

31 Gut microbiome; The Philippines 


\section{Introduction}

Polyparasitism, or infection with multiple parasite species, is highly prevalent within human populations of many economically poor countries. An estimated 2 billion people are infected worldwide with soil-transmitted helminths (STH) (Bethony et al., 2006), with individual species presenting a range of host pathologies while inhabiting different host tissue/organ sites (Fig. 1). STH share similar geographic ranges and similar infection pathways, with co-infections common (Gordon et al., 2017). Hookworms (Necator americanus and Ancylostoma spp.), whipworms (Trichuris trichiura) and roundworms (Ascaris lumbricoides) comprise the major clinically important STH species. STH transmission control is largely achieved by good hygiene practices, having access to latrines, and wearing shoes (Bieri et al., 2013; Campbell et al., 2014; McManus et al., 2014).

Parasitic helminths generally cause chronic disease, particularly in children, including growth stunting, reduced cognitive ability and anaemia in the case of hookworms and Schistosoma spp. (King, 2010; Parija et al., 2017). Hookworms are the only gut-resident helminths that penetrate the gut wall while feeding on host blood; the other gut-resident helminths attach to the intestinal lumen and impact on the mucosal immune system and homeostasis (Leung and Loke, 2013). Adult schistosomes dwell in the blood vessels of their human hosts. Intestinal helminth parasites and gut bacteria live in such close proximity in the intestine that it is likely considerable interaction between the two groups of organisms may lead to perturbations of the gut microbiome (Reynolds et al., 2015; Zaiss and Harris, 2016). There are some reports that helminth parasites increase host gut microbiome diversity (Cantacessi et al., 2014; Lee et al., 2014), whereas other studies showed no change in infected individuals (Cantacessi et al., 2014; Kay et al., 2015). How the presence of a single or multiple parasitic helminth species in the gut impacts this habitat, and whether the composition of the microbial population is affected, is largely unknown.

South East Asia (SEA) is a hot bed for STH infections, with approximately one-third of global cases occurring in this region (Jex et al., 2011). The Philippines is no exception, being highly endemic 
not only for STH but also S. japonicum and Taenia spp., often occurring as clinical co-infections (Gordon et al., 2015c). Here we report the gut microbiome of individuals with single or multiple parasitic helminth infections in a rural area of the Philippines (Gordon et al., 2015c). The study is timely as polyparasitism as a parameter has not been investigated when considering its potential impact on the gut microbiome of humans.

\section{Materials and methods}

\subsection{Ethics}

As previously described (Gordon et al., 2015b, 2015c), informed written consent was received from all participants and approval provided by the Ethics Committee of the Research Institute of Tropical Medicine (RITM), Manila, the Philippines, and the QIMR Berghofer Medical Research Institute (QIMRB) Human Research Ethics Committee, Australia (Approval Number: H0309-058 (P524)).

\subsection{Study area and design}

Stool samples were collected from two human cohorts in 2011 (Cohort-1; $n=161$ ), and 2015 (Cohort-2; $n=58$ ) from barangays in the municipalities of Palapag and Laoang in Northern Samar Province, the Philippines, as described (Fig. 2) (Supplementary Table S1) (Gordon et al., 2015a, 2015b; Olveda et al., 2017; Weerakoon et al., 2017). Of the total of 219 stool samples, 38 were helminth-negative by real time PCR (qPCR) and droplet digital PCR (ddPCR) (Supplementary Fig. S1); all helminth-negative individuals were from cohort 2. The ages of participants ranged from 4-72 years with the average age across the two cohorts being 33 years. Barangays in this study had been subjected in the past to annual mass treatment with praziquantel (PZQ) as part of the national schistosomiasis control program, although none of the selected study participants had been treated in the year prior to stool collection. School-aged children in the two cohorts were, at the time of stool 
collection, subjected to annual albendazole treatment for STH as part of the national control program in the Philippines (DepED, 2015a, b). Stool samples were stored in 80\% (v/v) ethanol for subsequent DNA isolation and molecular analysis.

DNA from subjects in cohort-1 was previously subjected to qPCR for $S$. japonicum (Gordon et al., 2015b) and multiplex qPCR for Ancylostoma spp.,Necator americanus, Ascaris lumbricoides, Taenia spp. and Trichuris trichiura (Gordon et al., 2015c). DNA from individuals in cohort-2 was previously used in a ddPCR for S. japonicum (Weerakoon et al., 2017). In the current study cohort-2 was subjected to a multiplex qPCR for Ancylostoma spp., Necator americanus, Ascaris lumbricoides, Taenia spp. and Trichuris trichiura as per cohort-1 (Gordon et al., 2015c). DNA samples from both cohorts $(n=219)$ were further subjected to microbiome profiling (Fig. 2).

DNA quality and quantity were determined using a NanoDrop 1000 (Thermo Scientific, Waltham, USA); DNA samples with quantity $<10 \mathrm{ng} / \mu 1$ and $260 / 280$ ratio of $<1.6$ or more than 2.1 were excluded. With cohort-1, 161 DNA samples were divided into single, dual, triple, quadruple, and quintuple helminth infection groups; then a set number of samples was randomly selected from each of these infection groups for microbiome analysis. For cohort-2, all DNA samples were analysed by multiplex qPCR after which 38 DNA samples negative for all helminth parasites and 20 helminthpositive DNA samples, selected based on age and gender matching with cohort-1, were submitted to microbiome analysis.

\subsection{DNA sequencing-based profiling of the gut microbiome}

The composition of the gut microbiome of all 219 cohort participants was profiled by $16 \mathrm{~S}$ rRNA amplicon sequencing (rrs) at the Australian Genome Research Facility (AGRF; Brisbane, Australia) following their standard protocols. The $r r s \mathrm{~V} 3$ and V4 hypervariable regions were amplified using primers 341F and 806R; Forward sequence: 5'-CCTAYGGGRBGCASCAG-3', Reverse sequence: 5'-GGACTACNNGGGTATCTAAT-3'. AmpliTaq gold 360 MasterMix (Life Technologies, Carlsbad, USA) was used for the first PCR assay and cycling conditions were as 
follows: initialisation at $95^{\circ} \mathrm{C}$ for 7 min followed by 30 cycles of $94^{\circ} \mathrm{C}$ for $45 \mathrm{~s}, 50^{\circ} \mathrm{C}$ for $60 \mathrm{~s}$ and $72^{\circ} \mathrm{C}$ for $60 \mathrm{~s}$, followed by a final extension of $72^{\circ} \mathrm{C}$ for $7 \mathrm{~min}$. A secondary PCR to index the amplicons was performed using TaKaRa Taq DNA polymerase (Clontech, Mountain View, USA). The resulting amplicons were measured by PicoGreen fluorometry (Invitrogen, Carlsbad, USA) and normalised. The equimolar pool was measured by qPCR (KAPA, Roche, Basel, Switzerland) followed by Illumina MiSeq sequencing with 2x300 bp paired-end chemistry.

Reads were assembled by aligning the forward and reverse reads using PEAR (V-0.9.5) (Zhang et al., 2014). Primers were identified, trimmed and $r r s$ amplicon sequences processed using Quantitative Insights into Microbial Ecology (QIIME-1.8) (Caporaso et al., 2010). Sequences were clustered into Operational Taxonomic Units (OTUs) using UCLUST on the basis of similarity to known bacterial sequences available in the GreenGenes database (v13.8, 97\% sequence similarity cut-off) and rarefied to a sequence depth of 28,700. Rare OTUs with $<5$ assigned amplicon sequences or $<0.0001$ fraction of total sequence reads were excluded and OTU reference sequences were aligned using pynast. The trimmed multiple alignment was then used to infer a phylogenetic tree (using fasttree) which, in turn, was used as input for UniFrac to estimate microbial beta diversity.

\subsection{Statistics}

SAS software (SAS Institute) was used for descriptive statistics and calculation of $95 \%$ confidence intervals (95\% CIs) of the parasitology data, while Calypso (version 8.54) (Zakrzewski et al., 2017) was used for mining the rrs microbiome dataset and for data visualisation. Rarefied and filtered OTU tables were uploaded to Calypso and square root transformed. To examine differences in the gut microbiota between infected and uninfected cohort participants, data were grouped as 'case' - parasite infected, and 'control' - negative for parasites. Data were further stratified in some instances to determine the effect of multiple parasite species (1-5) on bacterial diversity, and grouped as gut helminths (T. trichiura, hookworm, A. lumbricoides, Taenia spp.), S. japonicum only infections, and controls (negatives). Principal coordinate analysis (PCoA using weighted UniFrac distances) and Redundancy Analysis (RDA) were undertaken to assess whether variance in microbial 
133 community composition could be attributed to any of the study groups or clinical variables, such a 134 age and gender. Microbial species diversity was characterised using the Shannon and richness 135 diversity indices. Correlations with continuous variables (e.g. infection number) were calculated 136 using Spearman's rank order correlation. Individual taxa that were significantly differentially 137 abundant across study groups were identified by ANOVA and $P$ values were corrected for multiple 138 testing by false discovery rate (FDR). The significance level alpha was set to 0.05 .

139

\subsection{Data accessibility}

Raw data are available from Medeley Data. This includes a code book for annotation, an annotations excel workbook, and the raw OTU results in csv format (http://dx.doi.org/10.17632/59j46prhvf.1)

\section{Results}

\subsection{High prevalence of helminth parasites in the Palapag and Laoang endemic areas}

The majority of infected individuals $(70.32 \%)$ harboured at least two different helminth parasites with $7.31 \%$ (95\% CI: 3.83-10.78) being infected with at least five species (Supplementary Table S1). The highest prevalence was observed for S. japonicum (71.69\%; 95\% CI: 65.68-77.70) followed by $A$. lumbricoides $(48.86 \%$; 95\%CI: 42.19-55.53) and T. trichiura $(47.49 \%$; 95\% CI: 40.82-54.15) (Table 1).

\subsection{Significant changes seen in the gut microbiota between helminth-positive and helminth-} negative individuals

Microbial profiles were generally consistent with normal gut flora with high variability in the relative abundance of individual taxa between cohort participants (Supplementary Fig. S2). All microbiome data are provided in Supplementary Tables S2-S6.

RDA showed significant differences in the microbiome between helminth-parasitised individuals and controls $(P=0.001)$, while $S$. japonicum-only infections also showed significantly 
different microbiomes compared with individuals infected with intestinal helminths $(P=0.021)$ (Supplementary Fig. S3). The microbiome composition was considered for each helminth infection by a separate RDA; for example, $S$. japonicum infection, irrespective of infection with other species, were compared with individuals not infected with $S$. japonicum (including helminth-negative individuals). RDA clustering for S. japonicum, A. lumbricoides, T. trichiura, Taenia spp., and Ancylostoma spp. were all statistically significant $(P<0.05)$ (Fig. 3).

The most common bacterial phyla present in all samples analysed were Firmicutes and Bacteroidetes, followed by Actinobacteria (Supplementary Table S5). Archaea were significantly more abundant in helminth-positive individuals (cases, $n=181$; controls, $n=38, P \leq 0.01$, FDR=0.016) as were Firmicutes $(P \leq 0.01, \mathrm{FDR}=0.016)$ and Actinobacteria $(P \leq 0.001, \mathrm{FDR}=4.3 \mathrm{E}-08)$, while Bacteroidetes were significantly more abundant in helminth-negative individuals (controls, $n=38$, $P \leq 0.001, \mathrm{FDR}=4 \mathrm{E}-06)$.

\subsection{Helminth infections are associated with increased richness and diversity of the gut microbiota}

Bacterial richness was significantly higher $(P=6.96 \mathrm{e}-06)$ in individuals harbouring helminth parasites compared with those who did not (Fig. 4A), and significantly higher $(\mathrm{p} P=3.9 \mathrm{e}-07)$ in individuals with intestinal helminths $(n=167)$ compared with uninfected subjects $(n=38)$ or in those harbouring only $S$. japonicum $(n=14)$ (Fig. 4B). Bacterial richness increased significantly $(P=8.83 \mathrm{e}-$ 05, $\mathrm{R}=0.28$ ) as the number of infecting helminth species increased (Fig. 4C). Bacterial diversity, as measured by the Shannon Index, also increased in helminth-positive individuals $(P=0.0352$, Fig. 4A) compared with helminth-negative subjects, and in $S$. japonicum-only infected individuals $(P=0.0343$; Fig. 4B).

Bacterial richness was increased $(P=0.00366)$ in individuals harbouring $T$. trichiura irrespective of infection with other species, compared with individuals not infected with T. trichiura (Fig. 5A). Similarly, bacterial richness was significantly higher in $T$. trichiura-only infected individuals $(n=5)$ compared with helminth-negative controls $(n=38)(P=0.0111)$ (Fig. 5B). 


\subsection{Schistosoma japonicum infection has less impact on the gut microbiota composition than} intestinal helminth infection

We observed significant differences in the abundance of individual bacterial genera and species between helminth-infected subjects and uninfected controls (Supplementary Fig. S2 and Supplementary Fig. S4). When comparing S. japonicum-only $(n=14)$ infections with helminthnegative $(n=38)$ and intestinal helminth infections $(n=167)$, significant differences in abundance of certain bacteria were evident. However, in general, $S$. japonicum-only infected individuals had a similar microbiome as the intestinal helminth-infected subjects compared with those who were uninfected (Supplementary Fig. S5).

\subsection{Increased abundance of Lactobacillaceae in individuals with T. trichiura infection}

Lactobacillaceae were significantly more prevalent $(P=0.0034, \mathrm{FDR}=0.014)$ in parasitised individuals compared with those who were uninfected (Fig. 5). Regardless of infection with other helminths, Lactobacillus showed a higher relative abundance in T. trichiura-infected individuals ( $n=104)$, compared with $T$. trichuris-negative individuals $(n=115)$ (Fig. 5). There was no change in the relative abundance of the Lactobacillaceae between $T$. trichiura-only infected individuals $(n=5)$ compared with uninfected controls $(n=38)$.

Bacterial richness was increased $(P=0.00366)$ in individuals infected with $T$. trichiura, irrespective of infection with other species, compared with individuals not infected with $T$. trichiura (Fig. 5A). Similarly, bacterial richness was significantly higher in T. trichiura-only infected individuals $(n=5)$ compared with helminth-negative controls $(n=38)(P=0.0111)$ (Fig. 5B).

\subsection{Increased abundance of Faecalibacterium associated with any helminth infection}

There was an increase in Faecalibacterium abundance with increasing polyparasitism $(p=0.00044, \mathrm{R}=0.24)$ (Fig. 6A, Supplementary Fig. S6). Abundance of the genus Faecalibacterium was increased significantly $(P=0.038$; FDR 0.093$)$ in helminth-positive individuals compared with helminth-negative subjects (Fig. 6B). 
Comparisons were made between the cohort-1 helminth-positive individuals, cohort-2

209 helminth- positive individuals, and cohort-2 helminth-negative individuals to determine the impact

210 of batch effect and non-helminth infection status (Supplementary Fig. S7). In terms of richness, there

211 was a significant increase in diversity between cohort-1 and cohort-2 helminth-positive individuals 212 compared with those who were helminth-negative (Supplementary Fig. S7B). The Shannon Index 213 was not significant (0.053) but showed a trend in both cohorts towards increased diversity in 214 helminth-positive individuals compared with those who were helminth-negative.

\section{$215 \quad 4 . \quad$ Discussion}

This is the first known study to investigate the effect of helminth polyparasitism, a feature common in many rural communities of developing countries, on the human gut microbiome. Previous reports examining the gut microbiota and parasitism did not consider individuals infected with more than one helminth species, exemplified by recent studies with schistosome infections (Kay et al., 2015; Schneeberger et al., 2018). Another key limiting feature of previous studies was the use of insensitive microscopically-based diagnostic procedures to identify helminth infections. DNA diagnosis using multiplex qPCR is considerably more sensitive (Gordon et al., 2011, 2015c) and thus our data represent a more comprehensive overview of helminth parasites in this target study population.

The majority of published research on the gut microbiome has been undertaken in developed countries where the impact of helminth parasites, a major consideration here, is generally not a feature that is relevant or is considered. Our two study cohorts examined were from a rural, low socioeconomic area in the Philippines, and thus the microbiome profile might be expected to be quite different from that found in urban dwellers and residents from more economically developed countries (Mah et al., 2008; Tyakht et al., 2014; De Filippo et al., 2017). Accordingly, we found a 
232 of "rural microbiota species", examination of genus and family was used. We found a relatively high 233 abundance of Prevotella copri, a hallmark of rural infections and, in the Philippines, this species is 234 positively associated with a rural non-westernised diet (Nakayama et al., 2017). In addition, $P$. copri 235 was significantly associated with parasitic helminth infection $(P=0.0072)$ as reported previously for 236 S. haematobium infection and the microbiome (Kay et al., 2015) (Supplementary Table S2).

We found increasing numbers of helminth species co-infections correlated significantly with increased bacterial richness and diversity (Fig. 4C). Bacterial richness in S. japonicum-only infections ( $n=14)$ was reduced and comparable to that found in uninfected individuals (Fig. 4B), suggesting $S$. japonicum might have less of an effect on the gut microbiome than the STH. This could also be a 241 reflection of decreased richness due to a lower burden of helminths. We saw in this study an increase 242 in bacterial richness and diversity with increasing numbers of helminths as well. Intestinal 243 schistosomes impact physically on the host intestine only when their eggs traverse the gut lumen to 244 be excreted in faeces, although some become trapped in intestinal tissues, inducing mucosal 245 granulomatous inflammation (McManus et al., 2018). Previous studies examining the gut microbiota 246 and schistosome infection found significant differences between infected and uninfected individuals, 247 including differences in the microbiota composition in different age groups (Yatsunenko et al., 2012; 248 Kay et al., 2015). In contrast, we found no significant differences in our study by either age or gender 249 (data not shown). Ages in our study cohorts ranged from four to seventy-six, while differences had 250 previously been identified for children $<1$ year of age (Yatsunenko et al., 2012).

The increased bacterial diversity we report in STH-infected individuals compared with those who were uninfected, supports similar studies on the gut microbiota in rural Malaysians $(n=51)$ (Lee 253 et al., 2014) and Sri Lankans ( $n=76)$ (Jenkins et al., 2017), and highlights the impact of 254 gastrointestinal helminths on the microbiota diversity in parasitised individuals in both locations. The 255 Malaysian study also demonstrated that bacterial populations varied significantly between Malaysian 256 and New York City residents ( $p=0.01)$ (Lee et al., 2014). 
A low number of Proteobacteria is a marker of a healthy gut, and imbalances in the gut microbiota can be due to their increased abundance (Shin et al., 2015). In the current study we found no difference in the abundance of Proteobacteria in helminth-positive individuals compared with uninfected individuals. There was a slight decrease in Proteobacteria with increasing polyparasitism, 261 although this was not significant (Supplementary Fig. S6). The most abundant phyla present were the Firmicutes, followed by the Bacteroidetes, and then the Actinobacteria. There was a significant increase in the Firmicutes and Actinobacteria, but a significant decrease in Bacteroidetes in 264 parasitised subjects (Supplementary Table S2). Individual members of all these phyla can provide beneficial or elicit deleterious impacts on gut health; for instance a decrease in some Bacteroidetes and Firmicutes and an increase in Proteobacteria is commonly reported in Irritable Bowel Disease patients (Wexler, 2007). Members of the Firmicutes and Bacteroidetes are involved in a number of important metabolic processes within the intestine, specific examples being polysaccharide (fibre) 269 degradation and carbohydrate fermentation (Wexler, 2007) and they can influence the host immune system, benefiting themselves over other species and pathogens (Wexler, 2007). A reduction in Bacteroidetes abundance associated with parasitic infection has previously been documented (Andersen et al., 2016), suggesting that regulation of these bacteria by parasites occurs. It is noteworthy that the shifts in microbiota profiles likely reflect alterations in gut function and microbial 274 fermentation. We found an increase in the relative abundance of Firmicutes and Archaea with polyparasitism, most likely reflective of food digestion and anaerobic fermentation favouring the initial production of acetate, $\mathrm{CO}_{2}$ and hydrogen; and leading to methane and butyrate production. This contrasts with Bacteroidetes which produce relatively higher amounts of succinate and propionate. It has been hypothesised that parasites cause alterations within the gut microbiome to their own advantage; consequently, the higher levels of butyrate-producing Firmicutes might be more advantageous to gut parasites because butyrate has been shown to inhibit potentially deleterious 281 inflammatory responses within the intestine (Lepage et al., 2011; Riviere et al., 2016). 
individuals compared with uninfected controls, and an increase in abundance with increasing parasite diversity (Fig. 6 and Supplementary Fig. S6). Faecalibacterium is typically associated with a healthy microbiome in Western populations, and reduced numbers of these bacteria have been associated with Crohn's disease and other inflammatory conditions in the gut (Galecka et al., 2013; Cao et al., 2014).

Knowledge of Archaea, such as Euryarchaeota, within the gut is rudimentary, particularly in regards to their association with human disease (Koskinen et al., 2017). Certain Archaeal species are suggested to relate to Irritable Bowel Syndrome and colorectal cancer due to their role in the production of methane in the gut, with higher levels of methane linked to constipation-related disorders (Gaci et al., 2014). We found Euryarchaeota abundance significantly increased with increasing polyparasitism (Supplementary Fig. S6); this is to our knowledge the first time this observation has been made and may help explain further interactions within the gut. In contrast a study by Li et al. (2016) of goats infected with the intestinal nematode Haemonchus contortus displayed a lower abundance of Euryarchaeota in comparison to uninfected animals, suggesting this relationship is complex and may vary according to the host species infected and the infecting parasite species (Galecka et al., 2013).

An interesting finding in mouse models of Trichuris muris is the skewing of the immune response towards inflammation (Holm et al., 2015), corresponding with a decrease in bacterial diversity and an increase in Lactobacillus abundance. We found a similar trend in increasing abundance of Lactobacillus in subjects infected with T. trichiura (Fig. 5C). As there was marked polyparasitism in the study area, this could have been due to the effect of other helminth species and, indeed, there was a significant decrease in Lactobacillaceae in parasitised individuals compared with uninfected controls; Lactobacillus abundance was significantly associated with parasitised individuals (Supplementary Table S2). Thus we also examined individuals infected with only $T$. trichiura $(n=5)$ and compared them with parasite-negative controls. We observed a similar increased 
trend by the rank test, although there was no significant difference by T-test between the two groups

309 in Lactobacillus abundance, likely due to the small number of T. trichiura-only infected individuals

310 analysed. Mouse models have also shown a corresponding decrease in bacterial richness (Holm et al.,

311 2015) which we did not observe (Fig. 5B).

As a result of shared geographical endemic zones and infection pathways for the different species, polyparasitism, due to STH and S. japonicum, is widespread in the rural Philippines with single species infection being relatively rare (Gordon et al., 2015c). Accordingly, we examined stool samples from two cohorts from the same Palapag area, collected at different times, as few helminthnegative individuals were available in cohort-1 to act as controls. Utilising PCoA and RDA we found 317 a minimal batch effect between the two cohorts, and any differences reported here were not the result 318 of the different stool collection times; for example, significant findings of increased richness in 319 parasitised individuals were evident regardless of when the stool samples were collected 320 (Supplementary Fig. S7).

The interactions between bacteria, helminth parasites and their hosts are incredibly complex. Helminth infection generally leads to phases of both acute and chronic disease, and the entry routes of parasitic helminths into the host can lead to bacterial translocation which, in turn, can result in sepsis. By contrast helminth parasites are known to down-regulate inflammation and, as such, show potential for the treatment of inflammatory diseases. The current study focused on analysis of collected stool samples, which may not be fully representative of the entire gastrointestinal tract (GIT)

327 (Lavelle et al., 2015), as bacteria resident in the upper GIT were potentially missed. However, collecting such samples involves invasive procedures that are difficult to undertake in the field on a 329 large scale. Nevertheless, we clearly demonstrated that human infection with helminth parasites led 330 to an increase in bacterial richness and diversity, a feature often associated with a healthy gut. The 331 increased numbers of so called 'beneficial' bacteria, such as Faecalibacterium, we report in the 332 helminth-infected individuals points to a positive association leading to the concept that helminth 
333 parasites may in fact be beneficial commensal organisms.

334 Decreased bacterial richness and diversity is associated with dysbiosis and a range of diseases 335 associated with poor gut health. Our findings of increased richness and diversity in helminth 336 parasitized individuals may suggest a role for helminths, which are known to decrease inflammatory 337 responses, in good gut health.

\section{Acknowledgements}

340 We would like to acknowledge the excellent work of Tal Bavli (QIMRB, Australia) for 341 designing Fig. 1. The authors would like to acknowledge funding from UBS Optimus Foundation, 342 Switzerland and the Australian National Health and Medical Research Council for the cohort 343 sample collection in the Philippines and the Australian Infectious Diseases (AID) Research Centre 344 for seed funding for the microbiome 16S RNA analysis at the Australian Genome Research Facility 345 (AGRF). 


\section{References}

Andersen, L.O., Karim, A.B., Roager, H.M., Vigsns, L.K., Krogfelt, K.A., Licht, T.R., Stensvold, C.R., 2016. Associations between common intestinal parasites and bacteria in humans as revealed by qPCR. Eur J Clin Microbiol Infect Dis 35, (9) 1427-1431.

Bethony, J., Brooker, S., Alboico, M., Geirger, S.M., Loukas, A., Diemart, D., Hotez, P.J., 2006. Soil-transmitted helminth infections: ascariasis, trichuriasis, and hookworm. The Lancet 367, 1521-1532.

Bieri, F.A.M., Gray, D.J.P., Williams, G.M.P., Raso, G.P., Li, Y.-S.P., Yuan, L.P., He, Y.M.P.H., Li, R.S.B., Guo, F.-Y.B.A., Li, S.-M.B.A., McManus, D.P.D., 2013. Health-education package to prevent worm infections in Chinese schoolchildren. New Eng J Med 368, (17) 1603-1612.

Campbell, S.J., Savage, G.B., Gray, D.J., Atkinson, J.M., Magalhães, R.J.S., Nery, S.V., McCarthy, J.S., Velleman, Y., Wicken, J.H., Traub, R.J., WIlliams, G.M., Andrews, R.M., Clements, A.C.A., 2014. Water, sanitation, and hygiene (WASH): A critical component for sustainable soil-transmitted helminth and schistosomiasis control. PLoS Negl Trop Dis 8, (4) e2651.

Cantacessi, C., Giacomin, P., Croese, J., Zakrzewski, M., Sotillo, J., McCann, L., Nolan, M.J., Mitreva, M., Krause, L., Loukas, A., 2014. Impact of experimental hookworm infection on the human gut microbiota. J Infect Dis 210, (9) 1431-1434.

Cao, Y., Shen, J., Ran, Z.H., 2014. Association between Faecalibacterium prausnitzii Reduction and Inflammatory Bowel Disease: A Meta-Analysis and Systematic Review of the Literature. Gastroentero Res Pract 2014, 872725.

Caporaso, J.G., Kuczynski, J., Stombaugh, J., Bittinger, K., Bushman, F.D., Costello, E.K., Fierer, N., Pena, A.G., Goodrich, J.K., Gordon, J.I., Huttley, G.A., Kelley, S.T., Knights, D., Koenig, J.E., Ley, R.E., Lozupone, C.A., McDonald, D., Muegge, B.D., Pirrung, M., Reeder, J., Sevinsky, J.R., Turnbaugh, P.J., Walters, W.A., Widmann, J., Yatsunenko, T., Zaneveld, J., Knight, R., 2010. QIIME allows analysis of high-throughput community sequencing data. Nat Methods 7, (5) 335-336.

De Filippo, C., Di Paola, M., Ramazzotti, M., Albanese, D., Pieraccini, G., Banci, E., Miglietta, F., Cavalieri, D., Lionetti, P., 2017. Diet, Environments, and Gut Microbiota. A Preliminary Investigation in Children Living in Rural and Urban Burkina Faso and Italy. Front Microbiol 8, 1979.

DepED, 2015. Implementation of the national school deworming day, in: Education (Ed.), Division memorandum. DepED, Bohol, the Philippines, pp. 1-20.

DepED, 2015. Second Round of National School Deworming Day for School Year 2015-2016, in: Education (Ed.), Division memorandum. DepED, The Philippines, pp. 1-2.

Gaci, N., Borrel, G., Tottey, W., O'Toole, P.W., Brugere, J.F., 2014. Archaea and the human gut: New beginning of an old story. World J Gastroenterol20, (43) 16062-16078.

Galecka, M., Szachta, P., Bartnicka, A., Lykowska-Szuber, L., Eder, P., Schwiertz, A., 2013. Faecalibacterium prausnitzii and Crohn's disease - is there any connection? Pol J Microbiol 62, (1) 91-95.

Gordon, C.A., Gray, D.J., Gobert, G.N., McManus, D.P., 2011. DNA amplification approaches for the diagnosis of key parasitic helminth infections of humans. Mol Cell Probes 25, (4) 143152.

Gordon, C.A., Acosta, L.P., Gray, D.J., Olveda, R., Jarilla, B., Gobert, G.N., Ross, A.G., McManus, D.P., 2012. High prevalence of Schistosoma japonicum infection in carabao from Samar province, the Philippines: implications for transmission and control. PLoS Negl Trop Dis 6, (9) e1778.

Gordon, C.A., Acosta, L.P., Gobert, G.N., Jiz, M., Olveda, R.M., Ross, A.G., Gray, D.J., Williams, G.M., Harn, D., Yuesheng, L., McManus, D.P., 2015a. High prevalence of Schistosoma 

japonicum and Fasciola gigantica in bovines from Northern Samar, the Philippines. PLoS Negl Trop Dis 9, (2) e0003108.

Gordon, C.A., Acosta, L.P., Gobert, G.N., Olveda, D.M., Ross, A.G., Williams, G.M., Gray, D.J., Harn, D., Yuesheng, L., McManus, D.P., 2015b. Real-time PCR demonstrates high human prevalence of Schistosoma japonicum in the Philippines: implications for surveillance and control. PLoS Negl Trop Dis 9, (1) e0003483.

Gordon, C.A., McManus, D.P., Acosta, L.P., Olveda, R., Williams, M., Ross, A.G., Gray, D.J., Gobert, G.N., 2015c. Multiplex real-time PCR monitoring of intestinal helminths in humans reveals widespread polyparasitism in Northern Samar, the Philippines. Int J Parasitol 45, (7) 477-483.

Gordon, C.A., Kurscheid, J., Jones, M.K., Gray, D.J., McManus, D.P., 2017. Soil-transmitted helminths in tropical Australia and Asia. Tropical Medicine \& Infectious Disease 2, (4).

Holm, J.B., Sorobetea, D., Kiilerich, P., Ramayo-Caldas, Y., Estelle, J., Ma, T., Madsen, L., Kristiansen, K., Svensson-Frej, M., 2015. Chronic Trichuris muris Infection Decreases Diversity of the Intestinal Microbiota and Concomitantly Increases the Abundance of Lactobacilli. PLoS One 10, (5) e0125495.

Jenkins, T.P., Rathnayaka, Y., Perera, P.K., Peachey, L.E., Nolan, M.J., Krause, L., Rajakaruna, R.S., Cantacessi, C., 2017. Infections by human gastrointestinal helminths are associated with changes in faecal microbiota diversity and composition. PLoS One 12, (9) e0184719.

Jex, A.R., Lim, Y.A.L., M. Bethony, J., Hotez, P.J., Young, N.D., Gasser, R.B., 2011. Soiltransmitted helminths of humans in Southeast Asia-towards integrated control. Adv Parasitol 74, 231-265.

Kay, G.L., Millard, A., Sergeant, M.J., Midzi, N., Gwisai, R., Mduluza, T., Ivens, A., Nausch, N., Mutapi, F., Pallen, M., 2015. Differences in the Faecal Microbiome in Schistosoma haematobium Infected Children vs. Uninfected Children. PLoS Negl Trop Dis 9, (6) e0003861.

King, C.H., 2010. Parasites and poverty: the case of schistosomiasis. Acta Trop 113, (2) 95-104.

Koskinen, K., Pausan, M.R., Perras, A.K., Beck, M., Bang, C., Mora, M., Schilhabel, A., Schmitz, R., Moissl-Eichinger, C., 2017. First Insights into the Diverse Human Archaeome: Specific Detection of Archaea in the Gastrointestinal Tract, Lung, and Nose and on Skin. Mbio 8, (6).

Lavelle, A., Lennon, G., O'Sullivan, O., Docherty, N., Balfe, A., Maguire, A., Mulcahy, H.E., Doherty, G., O'Donoghue, D., Hyland, J., Ross, R.P., Coffey, J.C., Sheahan, K., Cotter, P.D., Shanahan, F., Winter, D.C., O'Connell, P.R., 2015. Spatial variation of the colonic microbiota in patients with ulcerative colitis and control volunteers. Gut 64, (10) 1553-1561.

Lee, S.C., Tang, M.S., Lim, Y.A.L., Choy, S.H., Kurtz, Z.D., Cox, L.M., Gundra, U.M., Cho, I., Bonneau, R., Blaser, M.J., Chua, K.H., Loke, P., 2014. Helminth colonization is associated with increased diversity of the gut microbiota. PLoS Negl Trop Dis 8, (5) e2880.

Lepage, P., Hasler, R., Spehlmann, M.E., Rehman, A., Zvirbliene, A., Begun, A., Ott, S., Kupcinskas, L., Dore, J., Raedler, A., Schreiber, S., 2011. Twin Study Indicates Loss of Interaction Between Microbiota and Mucosa of Patients With Ulcerative Colitis. Gastroenterology 141, (1) 227-236.

Leung, J.M., Loke, P., 2013. A role for IL-22 in the relationship between intestinal helminths, gut microbiota and mucosal immunity. Int J Parasitol 43, (3-4) 253-257.

Li, R.W., Li, W., Sun, J., Yu, P., Baldwin, R.L., Urban, J.F., 2016. The effect of helminth infection on the microbial composition and structure of the caprine abomasal microbiome. Sci Rep 6, 20606.

Mah, K.W., Sangsupawanich, P., Tunyapanit, W., van Bever, H., Shek, L.P., Chua, K.Y., Lee, B.W., 2008. Gut microbiota of children living in rural south Thailand and urban Singapore. Allergol Int 57, (1) 65-71.

McManus, D.P., Bieri, F.A., Li, Y.S., Williams, G.M., Yuan, L.P., Henglin, Y., Du, Z.W., Clements, A.C., Steinmann, P., Raso, G., Yap, P., Magalhaes, R.J., Stewart, D., Ross, A.G., 
Halton, K., Zhou, X.N., Olveda, R.M., Tallo, V., Gray, D.J., 2014. Health education and the control of intestinal worm infections in China: a new vision. Parasit Vectors 7, (1) 344.

McManus, D.P., Dunne, D.W., Sacko, M., Utzinger, J., Vennervald, B.J., Zhou, X.N., 2018. Schistosomiasis. Nat Rev Dis Primers 4, (1) 13.

Nakayama, J., Yamamoto, A., Palermo-Conde, L.A., Higashi, K., Sonomoto, K., Tan, J., Lee, Y.K., 2017. Impact of Westernized Diet on Gut Microbiota in Children on Leyte Island. Front Microbiol 8, (197).

Olveda, D.U., Inobaya, M., Olveda, R.M., Vinluan, M.L., Ng, S.-K., Weerakoon, K., McManus, D.P., Ramm, G.A., Harn, D.A., Li, Y., Lam, A.K., Guevarra, J.R., Ross, A.G., 2017. Diagnosing schistosomiasis-induced liver morbidity: implications for global control. Int J Infect Dis54, 138-144.

Parija, S.C., Chidambaram, M., Mandal, J., 2017. Epidemiology and clinical features of soiltransmitted helminths. Trop Parasitol 7, (2) 81-85.

Reynolds, L.A., Finlay, B.B., Maizels, R.M., 2015. Cohabitation in the Intestine: Interactions among Helminth Parasites, Bacterial Microbiota, and Host Immunity. J Immunol 195, (9) 4059-4066.

Riviere, A., Selak, M., Lantin, D., Leroy, F., De Vuyst, L., 2016. Bifidobacteria and ButyrateProducing Colon Bacteria: Importance and Strategies for Their Stimulation in the Human Gut. Front Microbiol 7.

Schneeberger, P.H.H., Coulibaly, J.T., Panic, G., Daubenberger, C., Gueuning, M., Frey, J.E., Keiser, J., 2018. Investigations on the interplays between Schistosoma mansoni, praziquantel and the gut microbiome. Parasit Vectors 11, (1) 168.

Shin, N.R., Whon, T.W., Bae, J.W., 2015. Proteobacteria: microbial signature of dysbiosis in gut microbiota. Trends Biotechnol 33, (9) 496-503.

Tyakht, A.V., Alexeev, D.G., Popenko, A.S., Kostryukova, E.S., Govorun, V.M., 2014. Rural and urban microbiota: To be or not to be? Gut Microbes 5, (3) 351-356.

Weerakoon, K.G., Gordon, C.A., Williams, G.M., Cai, P., Gobert, G.N., Olveda, R.M., Ross, A.G., Olveda, D.U., McManus, D.P., 2017. Droplet digital PCR diagnosis of human schistosomiasis: parasite cell-free DNA detection in diverse clinical samples. J Infect Dis. 216, 1611-1622.

Wexler, H.M., 2007. Bacteroides: the Good, the Bad, and the Nitty-Gritty. Clin Microbiol Rev 20, (4) 593-621.

Yatsunenko, T., Rey, F.E., Manary, M.J., Trehan, I., Dominguez-Bello, M.G., Contreras, M., Magris, M., Hidalgo, G., Baldassano, R.N., Anokhin, A.P., Heath, A.C., Warner, B., Reeder, J., Kuczynski, J., Caporaso, J.G., Lozupone, C.A., Lauber, C., Clemente, J.C., Knights, D., Knight, R., Gordon, J.I., 2012. Human gut microbiome viewed across age and geography. Nature 486, (7402) 222-227.

Zaiss, M.M., Harris, N.L., 2016. Interactions between the intestinal microbiome and helminth parasites. Parasite Immunol 38, (1) 5-11.

Zakrzewski, M., Proietti, C., Ellis, J.J., Hasan, S., Brion, M.J., Berger, B., Krause, L., 2017. Calypso: a user-friendly web-server for mining and visualizing microbiome-environment interactions. Bioinformatics 33, (5) 782-783.

Zhang, J., Kobert, K., Flouri, T., Stamatakis, A., 2014. PEAR: a fast and accurate Illumina PairedEnd reAd mergeR. Bioinformatics 30, (5) 614-620. 

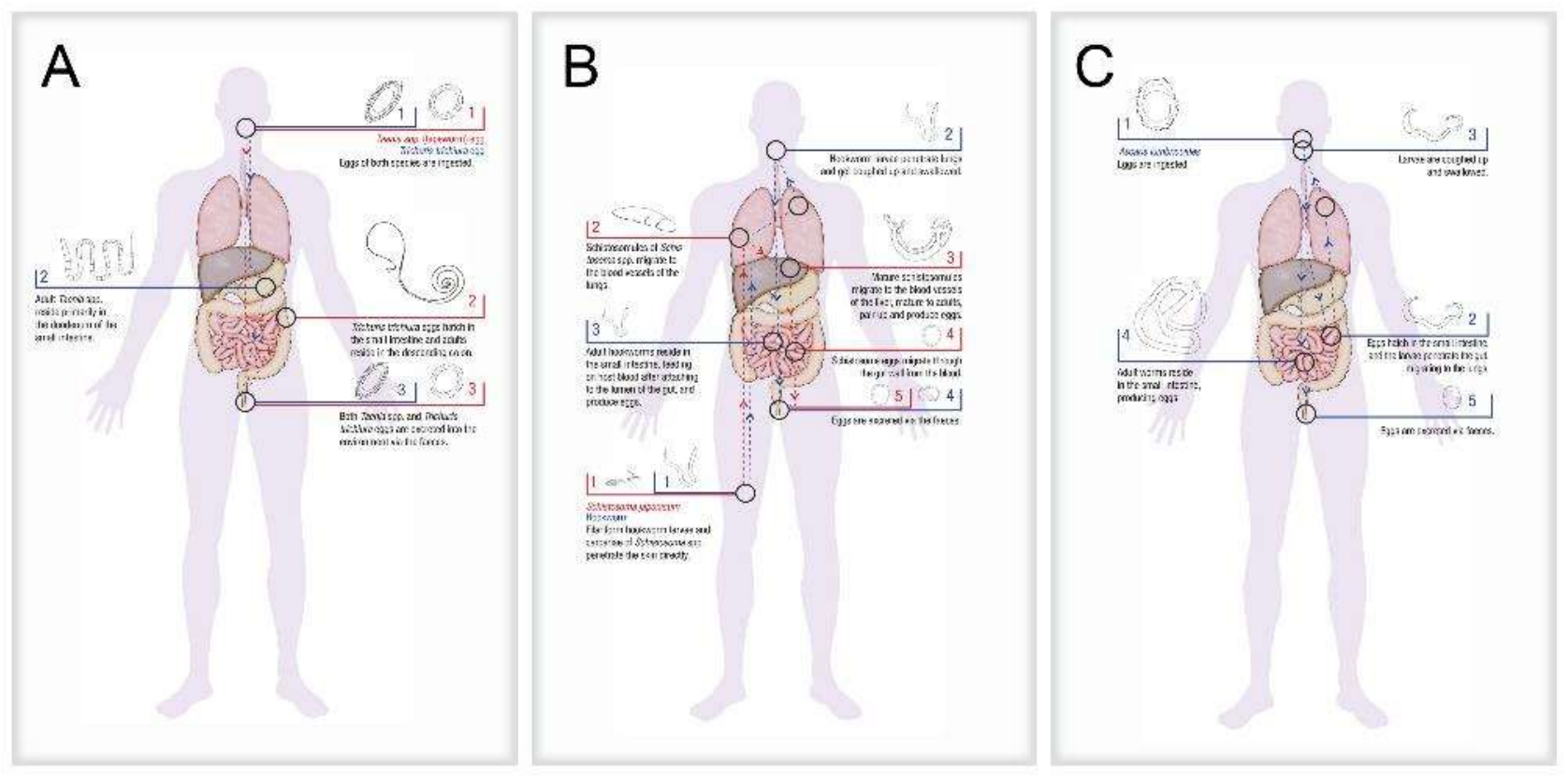

Fig. 1. Parasite lifecycles in the human host. (A) Lifecycle of Trichuris trichiura (blue; 1,2,3 on left) and Taenia spp. (red; 1,2,3 on right). In both species eggs are ingested (1) and eggs hatch in the gut. The adults of Taenia spp. live in the small intestine, particularly the duodenum (2) while T. trichiura adults reside in the large intestine, primarily the descending colon (2). Adults of both species produce eggs which are excreted in the faeces (3). (B) Lifecycle of Schistosoma japonicum (red; 1,2,3,5 on left, 4 on right) and Hookworm (blue; 1,2,4 on right, 3 on left). Larval forms of both species penetrate the skin directly (1) and migrate to the lungs. Hookworm larvae penetrate into the alveolar space and undergoing tracheal migration whereby the larvae are coughed up and swallowed (2). The hookworm larvae mature into adults in the gut, attaching to the lumen of the gut and feeding on host blood (3), and producing eggs which are excreted via the faeces (4). Schistosome larvae do not leave the blood vessels surrounding the lungs and are swept to the vessels around the liver where they mature and pair up (3), producing eggs which traverse the gut wall (4) to be excreted in the faeces (5). (C)

510 Lifecycle of Ascaris lumbricoides (blue). Mature eggs are ingested (1) and hatch in the gut. The larvae 511 penetrate the gut wall (2) and migrate to the lungs where they penetrate into the alveolar spaces and 512 undergo tracheal migration whereby they are coughed up and swallowed (3). Now mature, the adult 513 worms reside in the small intestine (4) and produce eggs which are excreted in faeces (5). 


\section{Collected in 2011}

Cohort 1

$$
\mathrm{N}=\mathbf{3 4 1 0}
$$

From 6 barangay in the municipality of Palapag, Northern Samar Province

$N=\mathbf{3 4 1 0}$

$\mathrm{N}=\mathbf{5 6 0}$

S. japonicum qPCR

completed

(Gordon, 2015a)

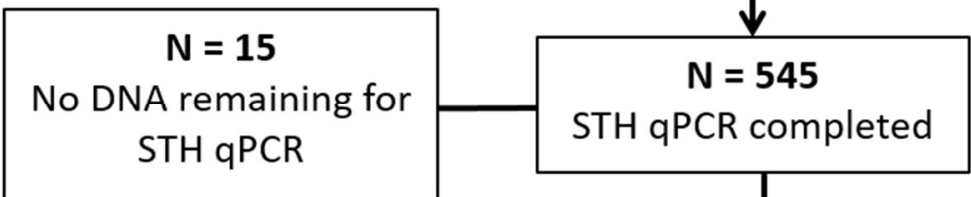

$\mathbf{N}=\mathbf{2 0}$

Parasite positive samples for 16S RNA microbiome diversity (Weerakoon, 2017)

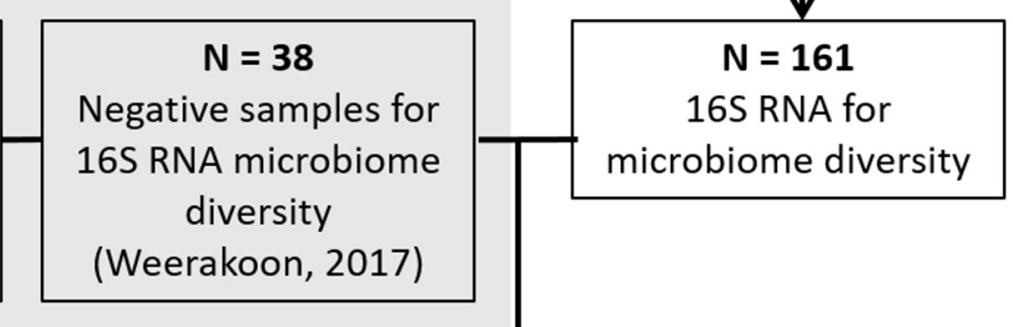

\section{Collected in 2015} Cohort 2

517 Fig. 2. Flow diagram showing initial cohort sample collection and real-time PCR (qPCR) analysis 518 through to the 16S RNA sequencing (Gordon et al., 2012; Gordon et al., 2015c; Weerakoon et al., 519 2017). STH, soil transmitted helminth. 

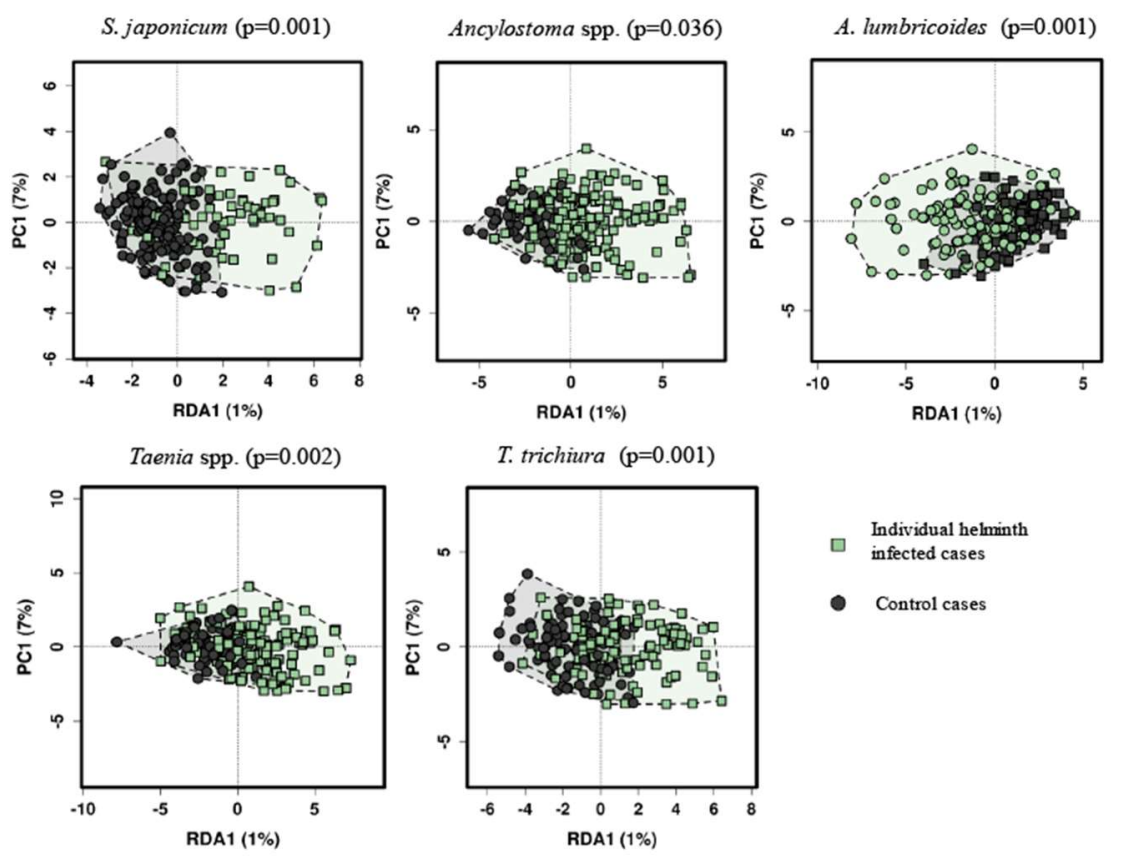

Individual helminth

$\square$ infected case

- Control cases

525 Fig. 3. Redundancy Analysis (RDA) of helminth-infected individuals in green and (remaining 526 individuals including negatives) in black. RDA was run on relative operational taxonomic unit (OTU) 527 abundance of infected cohort subjects with specific helminth species irrespective of infection with 528 other helminth species. 


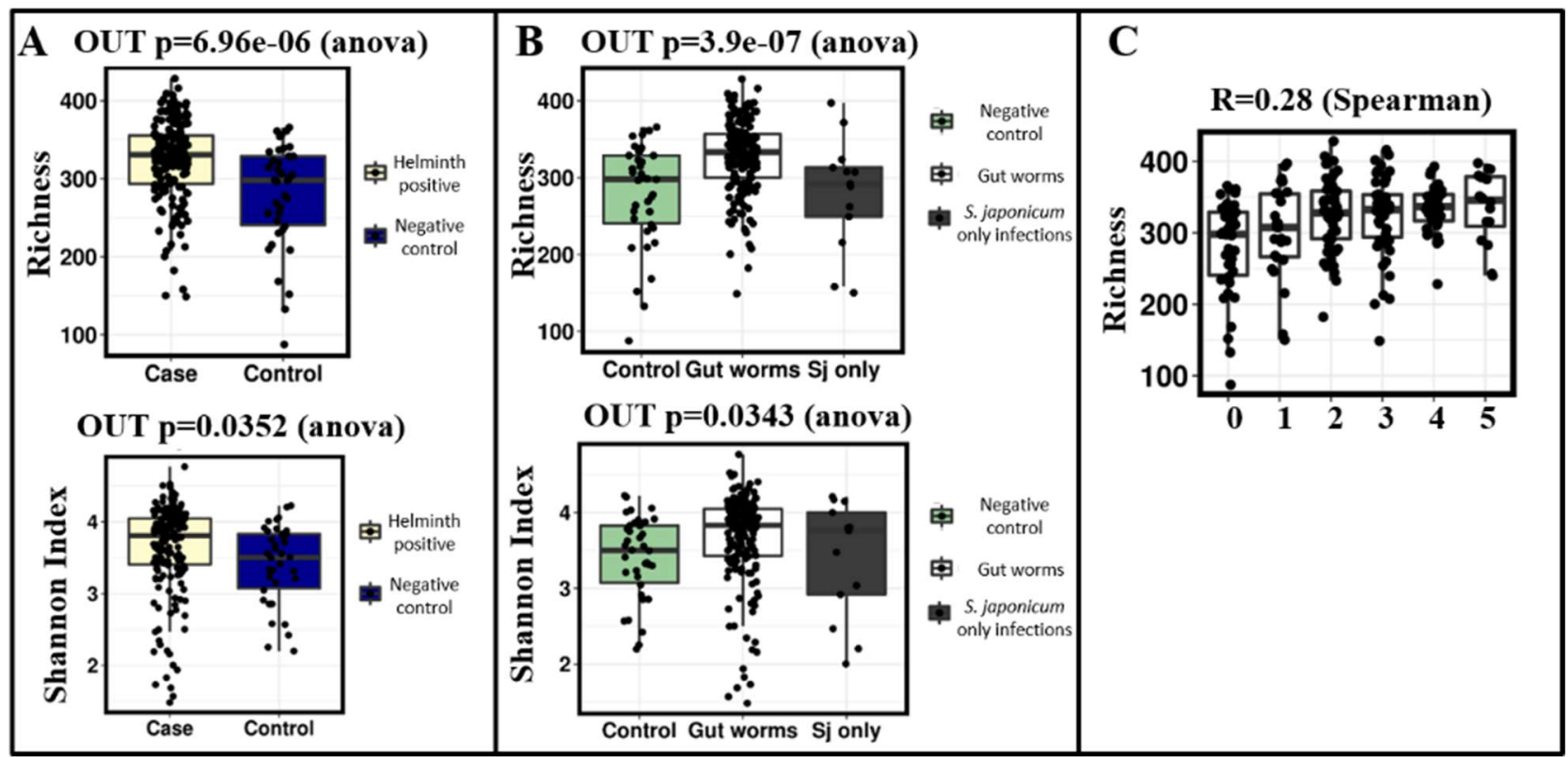

531 Fig. 4. Association between helminth infection status and microbial richness. (A) Comparison of

532 helminth-positive individuals and helminth-negative controls (uninfected individuals) considering

533 bacterial richness and diversity via the Shannon Index. (B) Comparison of helminth negative

534 individuals (negative controls) $(n=38)$ and individuals with intestinal helminths $(n=162)$, and those

535 infected with Schistosoma japonicum only $(n=14)$ considering bacterial richness and bacterial

536 diversity via the Shannon Index. (C) Comparison of bacterial richness with increasing number of 537 infections ranging from helminth-negative individuals $(n=38)$ to subjects with up to five co538 infections. OUT, operational taxonomic unit 
A

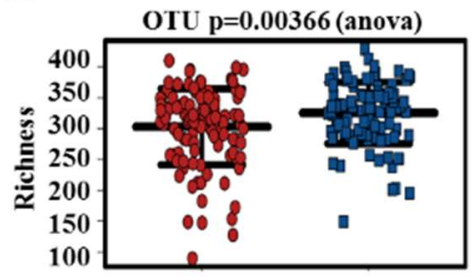

B

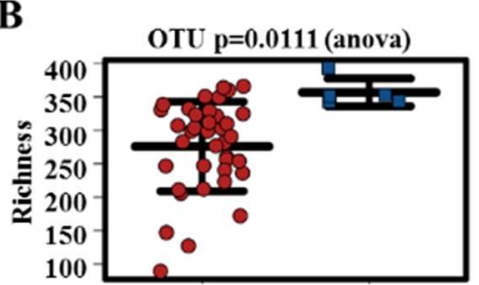

T-test

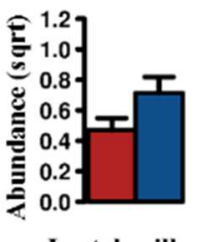

Lactobacillus

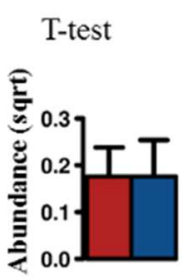

Lactobacillus
Rank test

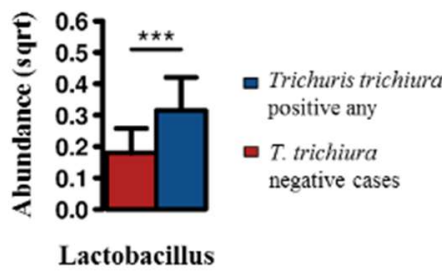

Rank test

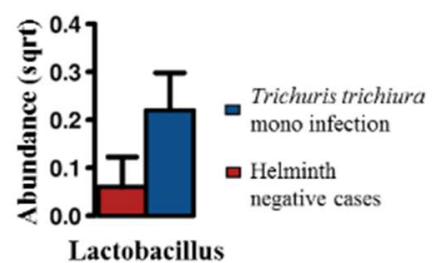

C T-test

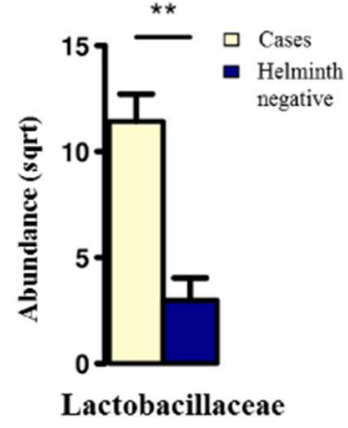

543 Fig. 5. Bacterial richness (total) and abundance of Lactobacillus when considering: (A) Trichuris 544 trichiura infection $(n=103)$, regardless of other infections, (blue, right) compared with $T$. trichiura 545 negatives $(n=116)$ (red, left) and (B) T. trichiura-only infections ( $n=5)$ (blue, right) compared with 546 helminth negatives (controls) $(n=38)$ (red, left). (C) The abundance of Lactobacillaceae in all 547 parasitised individuals (cases) $(n=181)$ versus controls $(n=38) . * P \leq 0.05 ; * * P \leq 0.01 ; * * * P \leq 0.001$. 


\section{T-test}
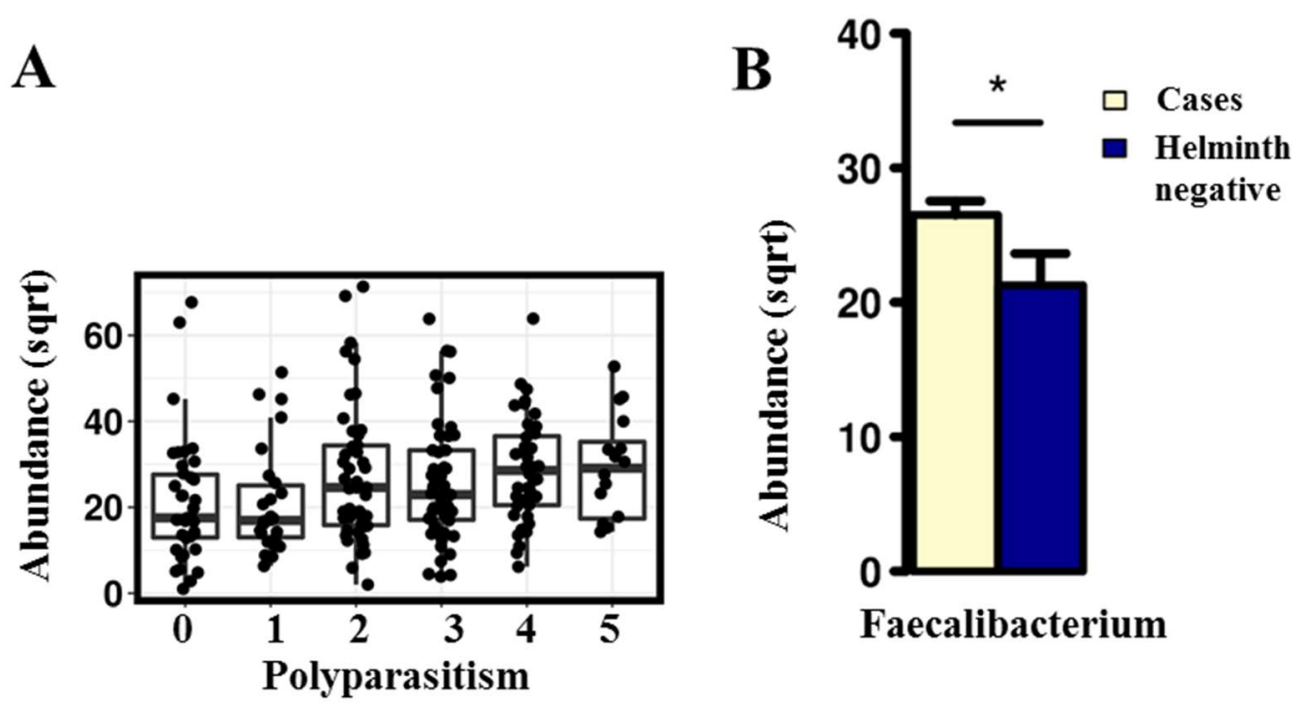

551 Comparison of Faecalibacterium abundance in control versus parasitised individuals. (A) 552 Comparison of abundance of Faecalibacterium with increasing numbers of infecting helminth 553 species ranging from no infections up to five co-infections ( $P=0.00044$, Spearman correlation). (B). 554 Relative abundance of Faecalibacterium in helminth-negative controls $(n=38)$ versus infected 555 individuals (cases; $n=181)(P=0.038$; FDR, false discovery rate $=0.093) . * P \leq 0.05$. 


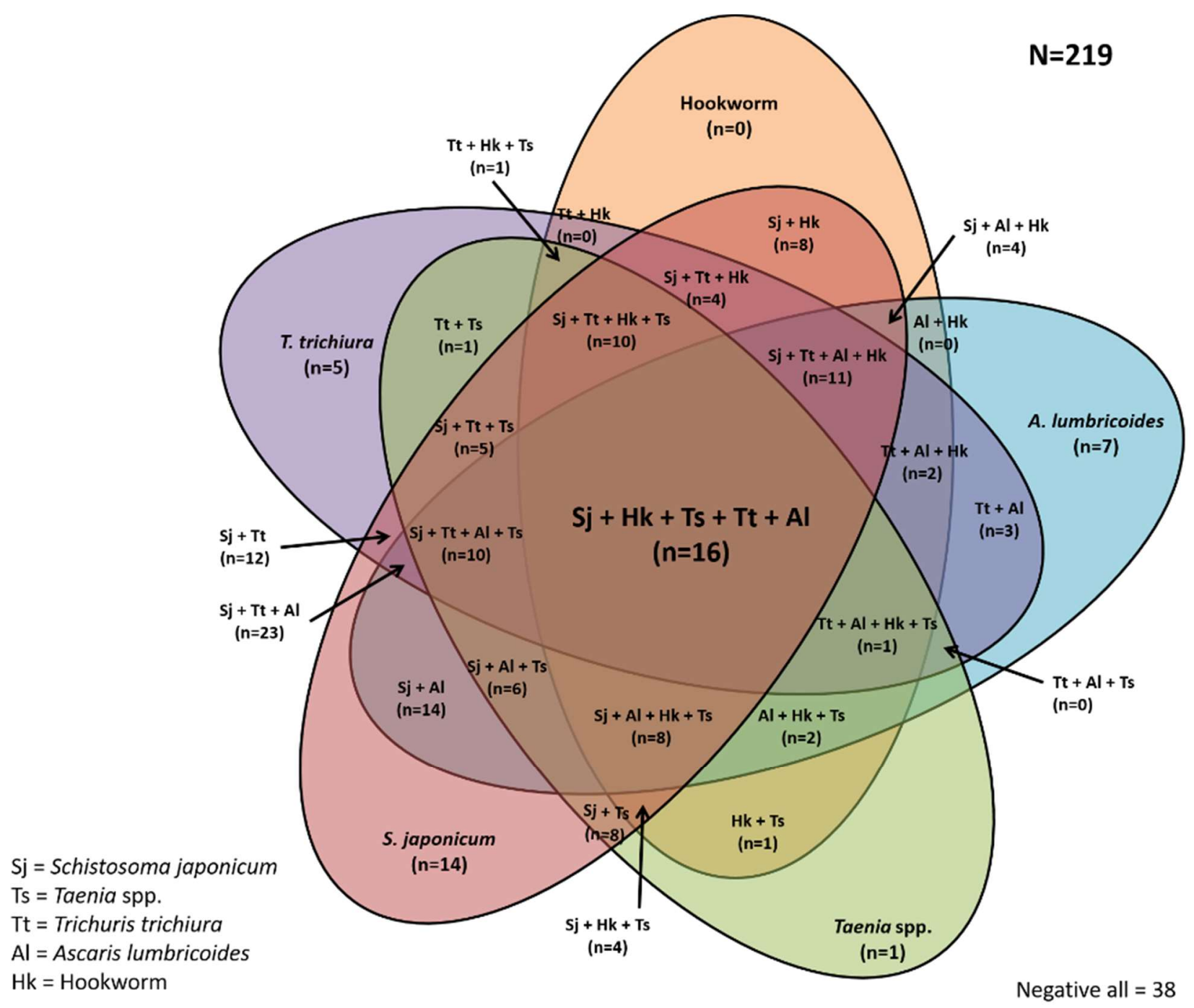

559 Supplementary Fig. S1. Venn diagram showing polyparasitism interactions for infected cohort 560 individuals. 


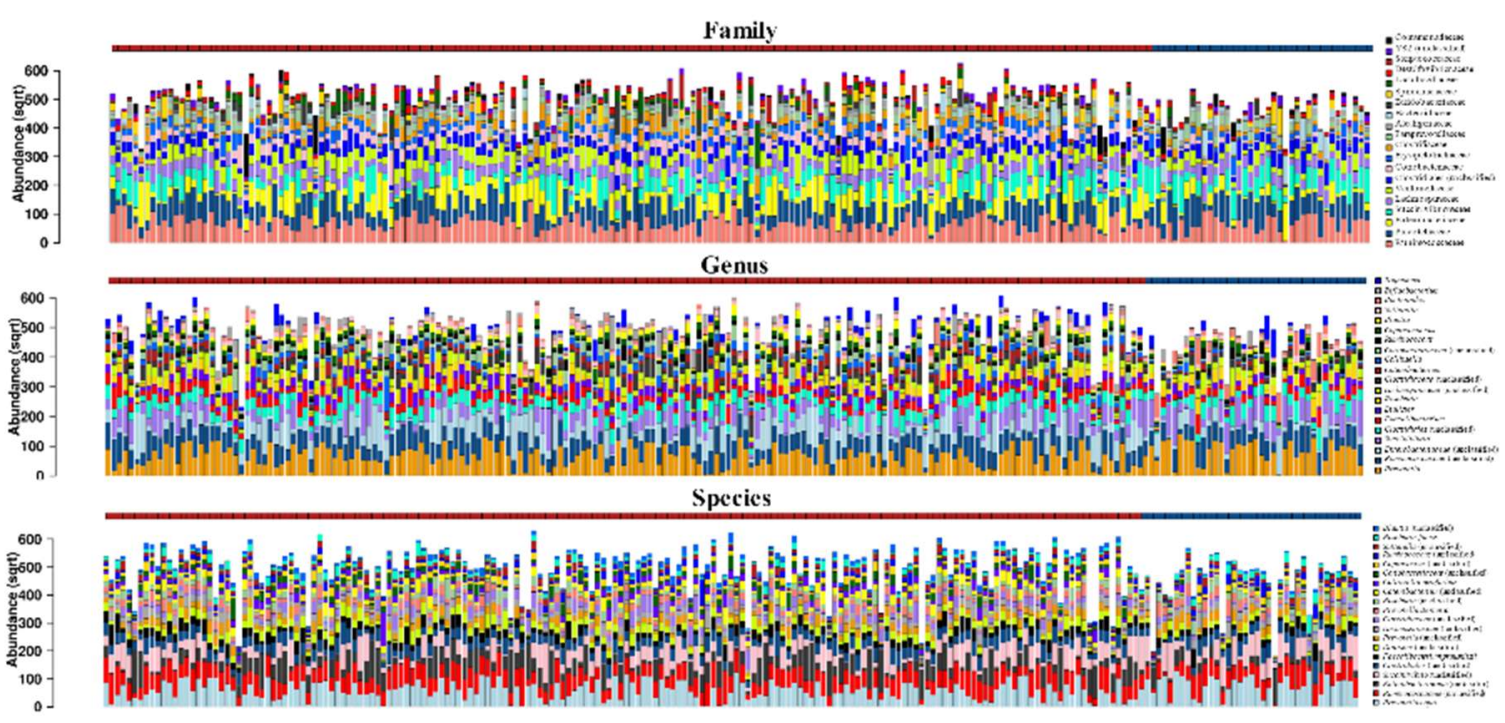

564 Supplementary Fig. S2. Abundance plots showing bacterial classifications of family, genus, and 565 species. The top line of each graph shows cases (red) and negative controls (blues). Figure legends 566 show colour coding for various bacterial families, genera, and species.
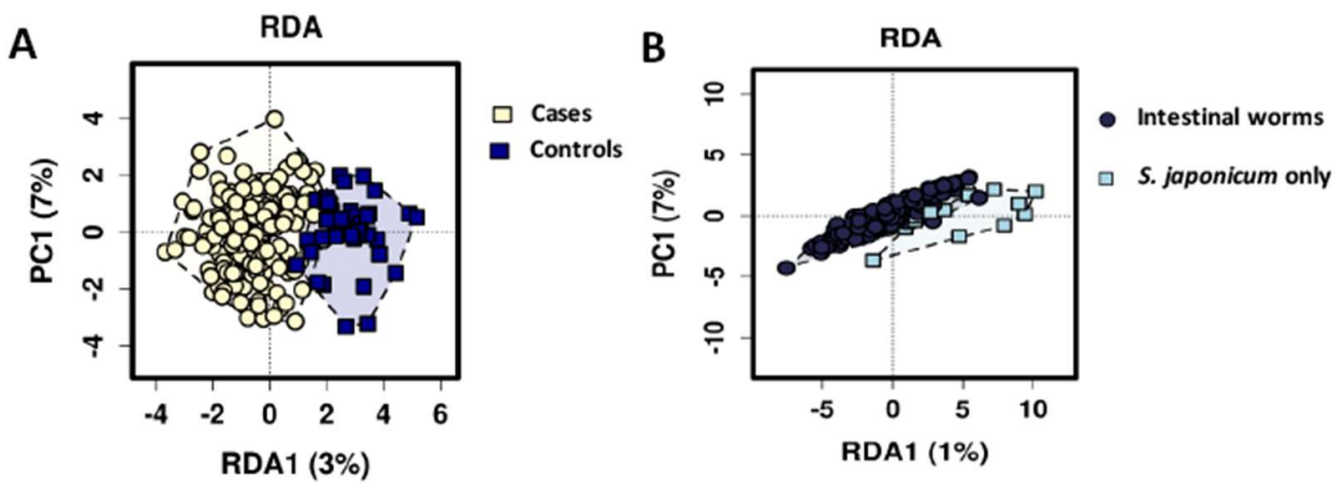

569 Supplementary Fig. S3. Supervised Redundancy Analysis (RDA) of: (A) helminth-positive cases 570 (cream) versus negative controls (blue) $(P=0.001)$; and $(B)$ intestinal helminths (dark blue) versus 571 Schistosoma japonicum-only (light blue) infections. RDA was run on relative OTU, operational 572 taxonomic unit abundances. 


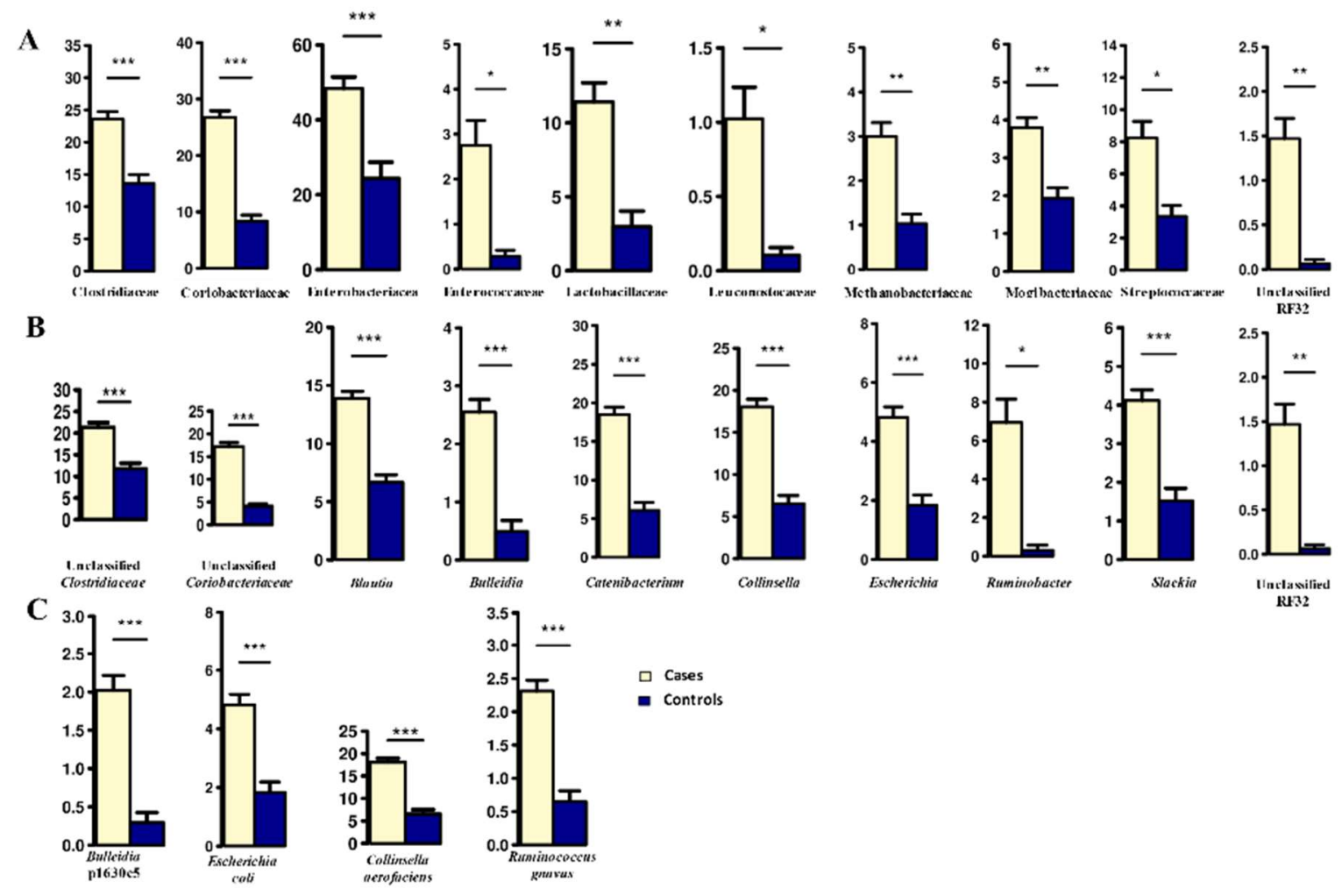

575 Supplementary Fig. S4. Abundance of significantly associated bacteria in helminth infection cases

576 by: (A) family; (B) genus; (C) species. Legend shows colour coding of cases (cream) versus negative 577 controls (blue) (Supplementary Table S2). ${ }^{*} P \leq 0.05 ; * * P \leq 0.01 ; * * * P \leq 0.001$. 

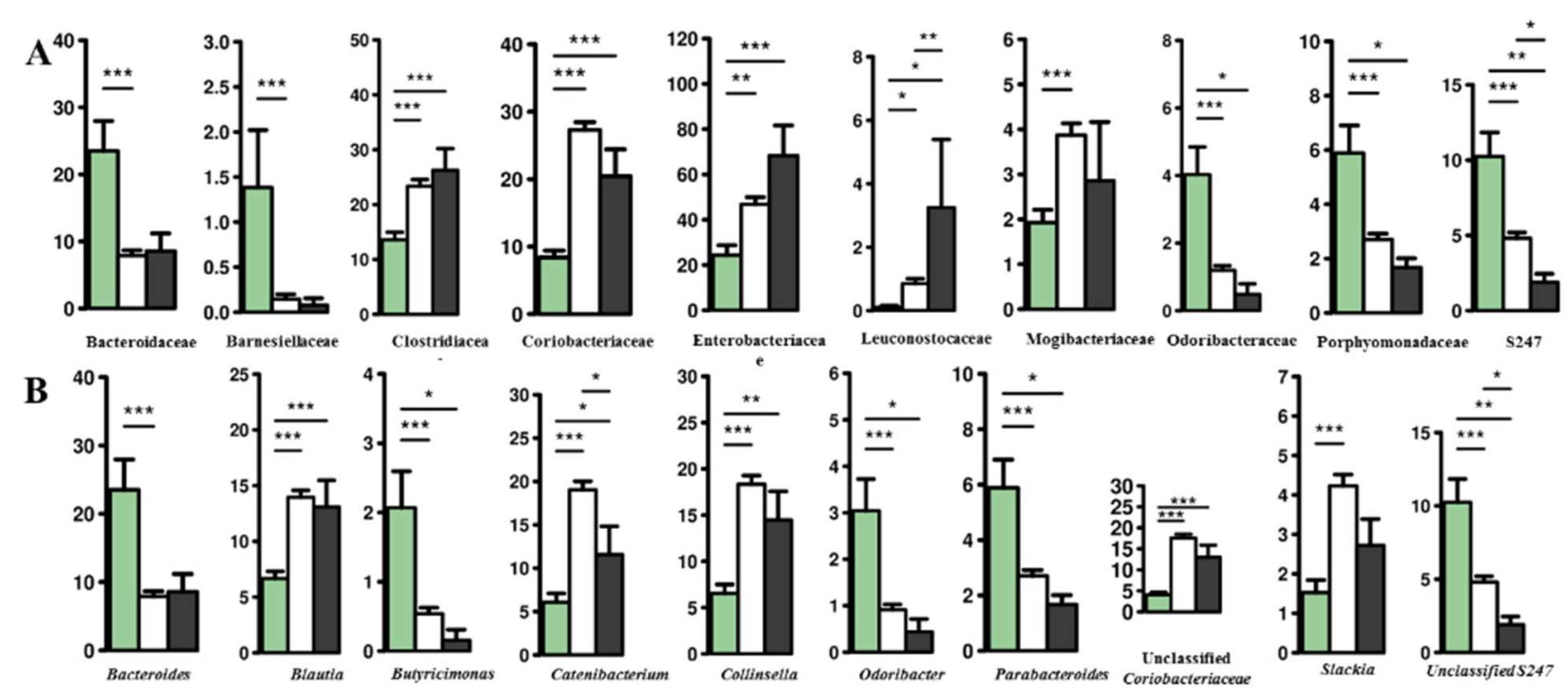

C
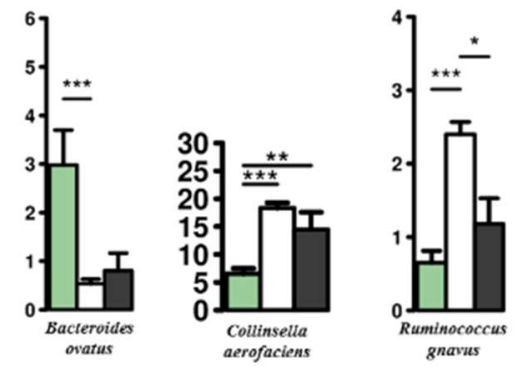

- S. japonicum only
$\square$ Intestinal worms
$\square$ Controls

581 Supplementary Fig. S5. Abundance of significantly associated bacteria in helminth infection cases:

582 (A) family; (B) genus; (C) species. Legend shows colour coding of Schistosoma japonicum only 583 infections (grey), intestinal helminths irrespective of S. japonicum infection (white), and negative 584 controls (green) (Supplementary Tables S2-S6). * $P \leq 0.05 ; * * P \leq 0.01 ; * * * P \leq 0.001$. 
A
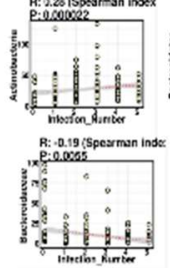

C

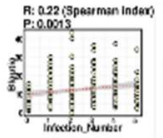

D

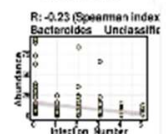

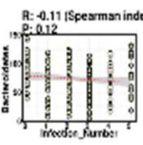
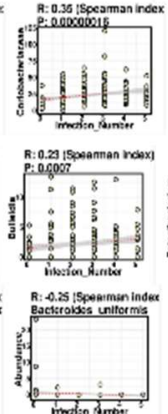
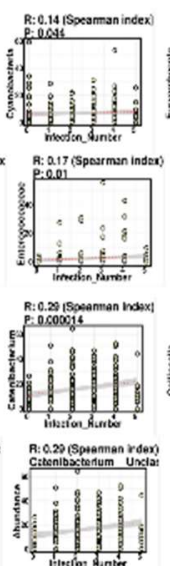
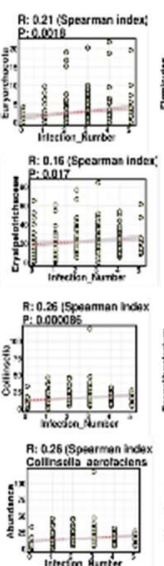
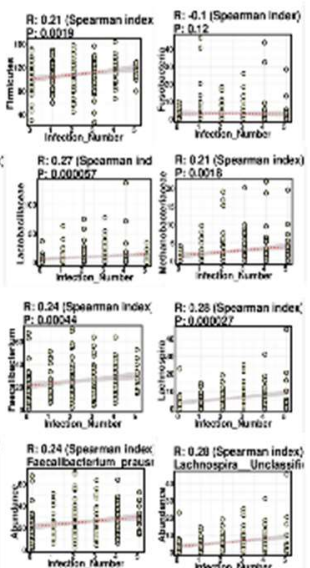
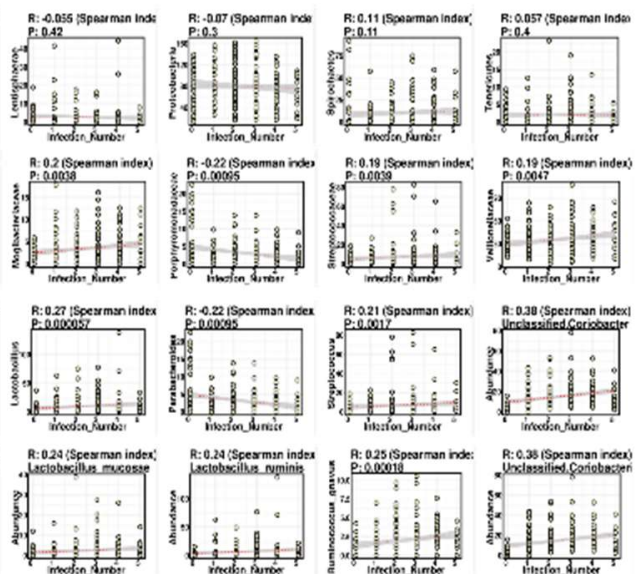

Supplementary Fig. S6. Significant associations of bacteria with increased complexity of helminth

species infection, showing phyla (A), family (B), genus (C), and species (D).
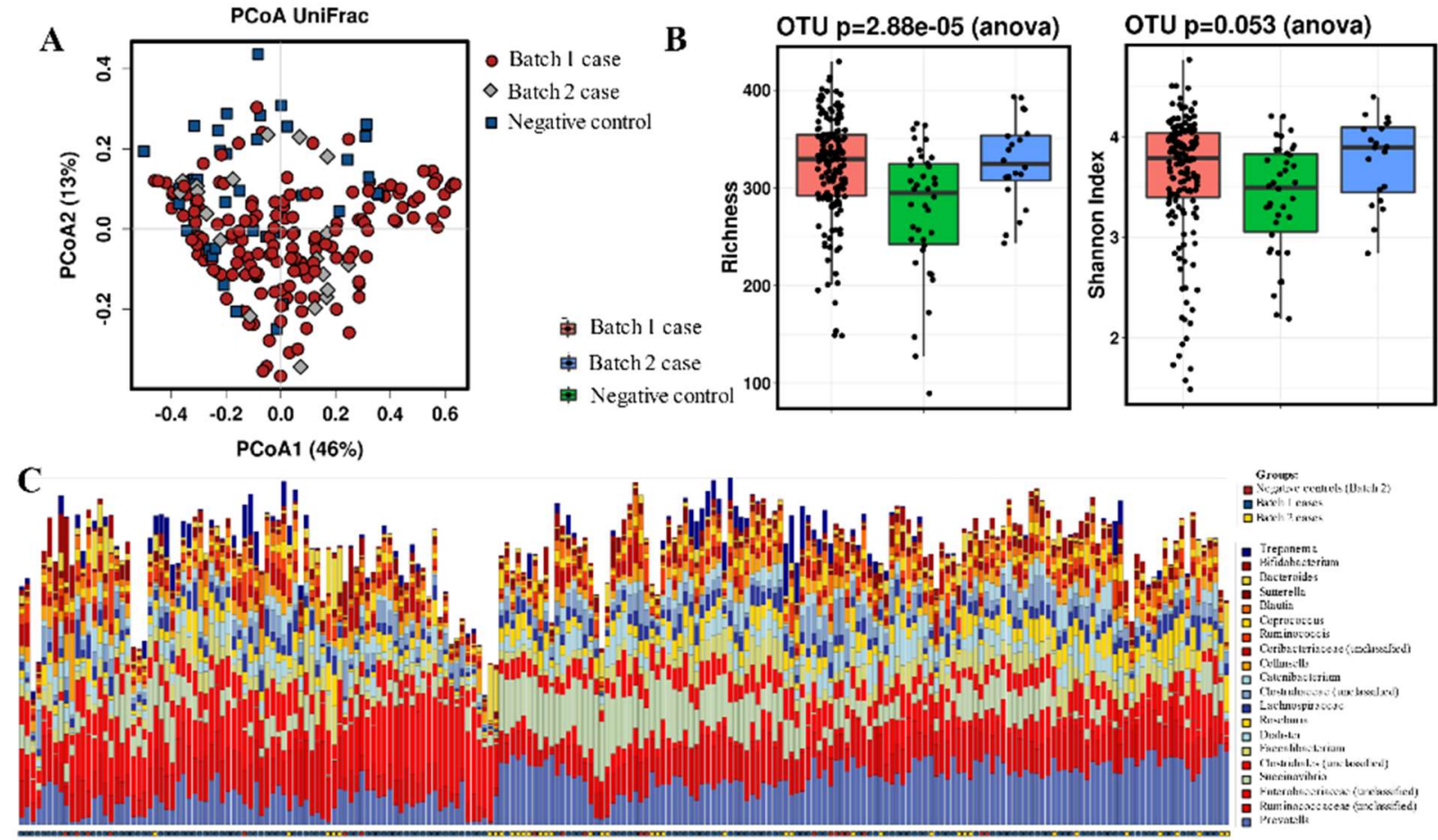

Supplementary Fig. S7. Exploring possible batch effect between the two cohorts. Cohort-1 faecal samples were collected in 2011, and cohort-2 in 2015 from the same area in the municipality of Palapag, Northern Samar Province, the Philippines. (A) Principal coordinate analysis (PCoA) of unweighted UniFrac distances comparing parasite-positive individuals in the cohort-1 collection 
596 (Batch 1 case, red) and in the cohort-2 collection (Batch 2 case, grey), compared with uninfected 597 (parasite-negative) individuals (negative control, blue). (B) Richness and Shannon index comparing 598 parasite-positive individuals in the cohort-1 collection (Batch 1, pink) and in the cohort-2 collection 599 (Batch 2, blue), compared with uninfected (parasite-negative) individuals (Batch 2, green). (C) 600 Overview of bacterial genus composition from the Municipality of Palapag, Northern Samar 601 Province, the Philippines study area. The bottom column shows collection times as per the key with 602 the cohort-1 collection (Batch 1, blue) and the cohort-2 collection (Batch 2, red), compared with 603 uninfected (parasite-negative) individuals (Batch 2, yellow). OTU, operational taxonomic unit 604 605 606

Table 1. Infection prevalence of helminth parasites in the two cohorts

\begin{tabular}{lccc}
\hline Species & No. Positive & Prevalence (\%) & 95\% CI \\
\hline Schistosoma japonicum & 157 & 71.69 & $65.68-77.70$ \\
Trichuris trichiura & 104 & 47.49 & $40.82-54.15$ \\
Ascaris lumbricoides & 107 & 48.86 & $42.19-55.53$ \\
Hookworm & 72 & 32.88 & $26.61-39.15$ \\
Taenia spp. & 74 & 33.79 & $27.48-40.10$ \\
Negative & 38 & 17.35 & $12.30-22.41$ \\
\hline 95\% CI, 95\% confidence interval. & & &
\end{tabular}

